\title{
Reference publication year spectroscopy (RPYS) of Eugene Garfield's publications
}

\author{
Lutz Bornmann $^{1}$ - Robin Haunschild ${ }^{2}$ Loet Leydesdorff ${ }^{3}$
}

Received: 26 June 2017/Published online: 5 December 2017

(C) The Author(s) 2017. This article is an open access publication

\begin{abstract}
Which studies, theories, and ideas have influenced Eugene Garfield's scientific work? Recently, the method reference publication year spectroscopy (RPYS) has been introduced, which can be used to answer this and related questions. Since then, several studies have been published dealing with the historical roots of research fields and scientists. The program CRExplorer (http://www.crexplorer.net) was specifically developed for RPYS. In this study, we use this program to investigate the historical roots of Eugene Garfield's oeuvre.
\end{abstract}

Keywords Cited references - Reference publication year spectroscopy $\cdot$ Eugene Garfield · Historical roots · RPYS · Pioneer · Bibliometrics

\section{Introduction}

Bibliometrics has become a central component of research evaluation. Field-normalized indicators are used to assess the scientific performance of institutions and countries. Individual researchers are well informed about their $h$ index. The development to this

Lutz Bornmann

bornmann@gv.mpg.de

Robin Haunschild

R.Haunschild@fkf.mpg.de

Loet Leydesdorff

loet@leydesdorff.net

1 Division for Science and Innovation Studies, Administrative Headquarters of the Max Planck Society, Hofgartenstr. 8, 80539 Munich, Germany

2 Max Planck Institute for Solid State Research, Heisenbergstr. 1, 70569 Stuttgart, Germany

3 Amsterdam School of Communication Research (ASCoR), University of Amsterdam, PO Box 15793, 1001 NG Amsterdam, The Netherlands 
prominence of bibliometrics had not been possible without the groundbreaking work of Eugene Garfield (EG). EG conceptualized citation indexing in science and published the underlying concept in Science (Garfield 1955). His invention of the index "revolutionized the world of scientific information and made him one of the most visionary figures in information science and scientometrics" (van Raan and Wouters 2017). In the 1960s, he started the professional use of bibliometrics by founding the Institute for Scientific Information (ISI) (now Clarivate Analytics, see clarivate.com). He developed the Science Citation Index, the Social Sciences Citation Index, and the Arts and Humanities Citation Index. These indexes are predecessors of the Web of Science (WoS) which is well-known today to scholars (Small 2017; Wouters 1999b). During EG's lengthy career, he published more than 1500 publications (see www.researcherid.com/rid/A-1009-2008).

In this study, we are interested in the roots of EG's oeuvre. Which studies, theories, and ideas have influenced EG's own scientific work? Marx et al. (2014) introduced reference publication year spectroscopy (RPYS), which can be used to answer this and related questions. In recent years, several studies have been published dealing with the historical roots of research fields. For example, Hou (2017) deals with the historical roots of citation analysis in a recent study. Thor et al. (2016a) developed the CRExplorer-a program specifically designed for RPYS (http://www.crexplorer.net). In the study, we use this program to investigate the historical roots of EG's oeuvre.

\section{Dataset}

The study is based on papers published by EG. We used his ResearcherID to collect his publications and added some further publications which are not covered by the ResearcherID. The dataset consists of $n=1558$ papers published between 1954 and 2016 (containing 15,890 cited references, CRs). The papers have been retrieved from the WoS. The downloaded files were imported in the CRExplorer for further processing (Thor et al. 2016a, b). The removing, clustering, and merging functionalities of the CRExplorer have been used to clean the CR dataset, especially for variants of the same CR. The clustering and merging of variants (using volume and page number information) lead to a slightly reduced set of CRs $(n=15,850)$. A further 199 CRs were deleted because the bibliographic information was unprecise (for example, no reference publication year, RPY, was provided). We formed two datasets from this initial set for the RPYS:

(1) Dataset including self-citations by EG We deleted those CRs which have occurrences of less than $10 \%$ in a single year (i.e. the share of occurrences for a CR among the occurrences of all CRs in one year is less than 10\%). This step leads to the first set of $328 \mathrm{CRs}$. Note that this also removes two of the most referenced CRs with $n=100$ and $n=97$ occurrences as their share of occurrences in their RPYs is 9.9 and $8.3 \%$, respectively. However, there are a number of CRs which appeared in Current Contents without a volume number; so they do not provide useful bibliometric information (see below).

(2) Dataset without self-citations We deleted all self-cited publications $(n=1283)$. Since the CR data in the WoS only contains the first author name, the identification of self-citations is restricted to this first name. As above, we deleted those CRs which have occurrences of less than $10 \%$ in a single year (see above). This step results in the second final set of $316 \mathrm{CRs}$. Although the difference between data set 1 and data set 2 (12 CRs) appears to be rather small at a first glance, the spectrogram 
changes significantly. Most self-cited CRs $(n=1271)$ are removed due to the occurrence threshold of $10 \%$ in a single year.

\section{Results}

The 1558 papers authored by EG were published in 125 different journals, but $68.2 \%$ of his papers were published in Current Contents. In the 1950s, Current Contents has been founded by EG as a rapid alerting service (Small 2017). The first subject edition covered biology and medicine. Other subject editions were added later, and the original version was renamed into Current Contents Life Sciences. Initially, Current Contents consisted of printed versions of reproduced title pages of major peer-reviewed journals. An author index with addresses and a subject index were provided as well. This indicates that EG's publications in Current Contents have a status different from papers in peer-reviewed journals. Similarly, EG founded The Scientist as a professional magazine for scientists in 1986. Although this journal was also incorporated into the Science Citation Index, it does not contain references and is thus only registered for being cited; including a Journal Impact Factor (JIF) of 0.369 in 2015.

Table 1 shows the journals in which EG has published at least 10 papers. The journals shown contain $88.6 \%$ of EG's publication output.

EG has published most of his papers $(80.9 \%)$ between 1970 and 1990. During these two decades, he has published 60 papers per year on average. However, $77.8 \%$ of the papers during this time frame were published in Current Contents. The unusual distribution of EG's papers across journals and publication years shows that EG has been an unusual scientist preferring a journalistic medium.

Without the publications in Current Contents and The Scientist, EG's oeuvre is 257 publications in scholarly journals containing 2244 references. Figure 1 compares the citations to EG's full and scholarly oeuvre. The two papers in Science of 1955 and 1972 are heavily cited. The erratic blue-colored curve is mainly citation of Current Contents and to a large extent self-citation. The peak in 2006 is an article in JAMA entitled "The history and meaning of the journal impact factor".

Table 1 Journals with at least 10 papers published by EG

\begin{tabular}{lc}
\hline Journal & Number of papers \\
\hline Current Contents & 1063 \\
Scientist & 148 \\
Current Contents Life Sciences & 88 \\
Journal of Information Science & 13 \\
Scientometrics & 12 \\
Nature & 12 \\
Journal of Chemical Documentation & 12 \\
Journal of the American Society for Information Science & 11 \\
Abstracts of Papers of the American Chemical Society & 11 \\
Science & 10 \\
\hline
\end{tabular}




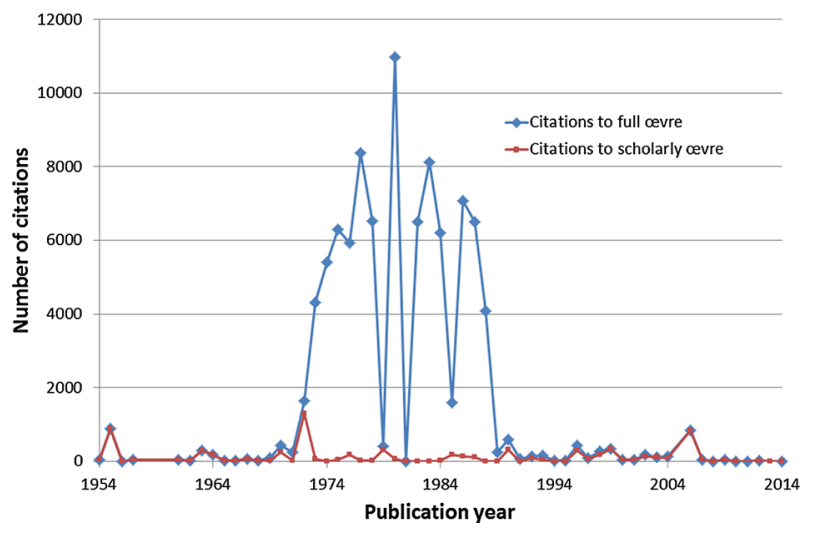

Fig. 1 Number of citations to EG's full and scientific oeuvre

The spectrogram of the number of CRs and the 5-year median deviations for data set 1 is shown in Fig. 2. The spectrogram features five large peaks at RPYs 1955, 1971-1975 (with a shoulder), 1978, 1985, and 1987/1988. Table 3 lists the CRs which are mainly responsible for these five peaks. In addition to the bibliographic information, the table provides the number of occurrences (in other words, how often the publication has been cited by EG) in absolute terms. It is clearly visible that the result is dominated by selfcitations. EG seems to have frequently based his newer publications on the footing of his earlier publications. EG was a pioneer and there were no other shoulders than his own, on which he could base his research. Similarly to EG's publication output, also his referencing style is skewed.

Nine of the eleven CRs in Table 2 appeared in Current Contents. Unfortunately, no issue or volume numbers are provided in most cases. In 1978, EG has published 52 papers in Current Contents with the beginning page number 5-in practice, this is the opening page. Therefore, it is not possible to provide more information about most CRs in Table 2 by bibliometric means; one would have to retrieve and read these contributions. Furthermore, it is unclear which of the volumes/issues was meant by the reference. However, the CR with RPY 1985 (Garfield, E., 1985, Current Contents, V43, P3) is about "uses and misuses of citation frequency". The first and the third CRs are two papers in Science. The 1955 paper introduces the concept of the SCI; the 1972 paper is the locus classicus for explaining the journal impact factor. These are the two most cited papers of EG. They both introduce a major innovation.

In order to receive hints on publications by other researchers which might have influenced EG's research, we performed a second RPYS after excluding (first author) selfcitations (data set 2).

The spectrogram of the number of CRs (blue line) and the five-year median deviations (black line) is shown in Fig. 3. The main difference between Figs. 2 and 3 is that 12 additional self-cited CRs (after applying the 10\% threshold criterion) remain in data set 1 but are removed from data set 2 . These 12 additional self-cited CRs are mainly responsible for the shape of the spectrogram from data set 1 (see Fig. 2) in comparison to the shape of the spectrogram from data set 2 (see Fig. 3). The spectrogram in Fig. 3 shows many small peaks and only one distinguished larger peak at the RPY 1951. The reference to Lowry et al. (1951) is an example used in bibliometrics to discuss non-justified extremely high citations (TC $>300,000)$. This is the first CR in Table 3. 


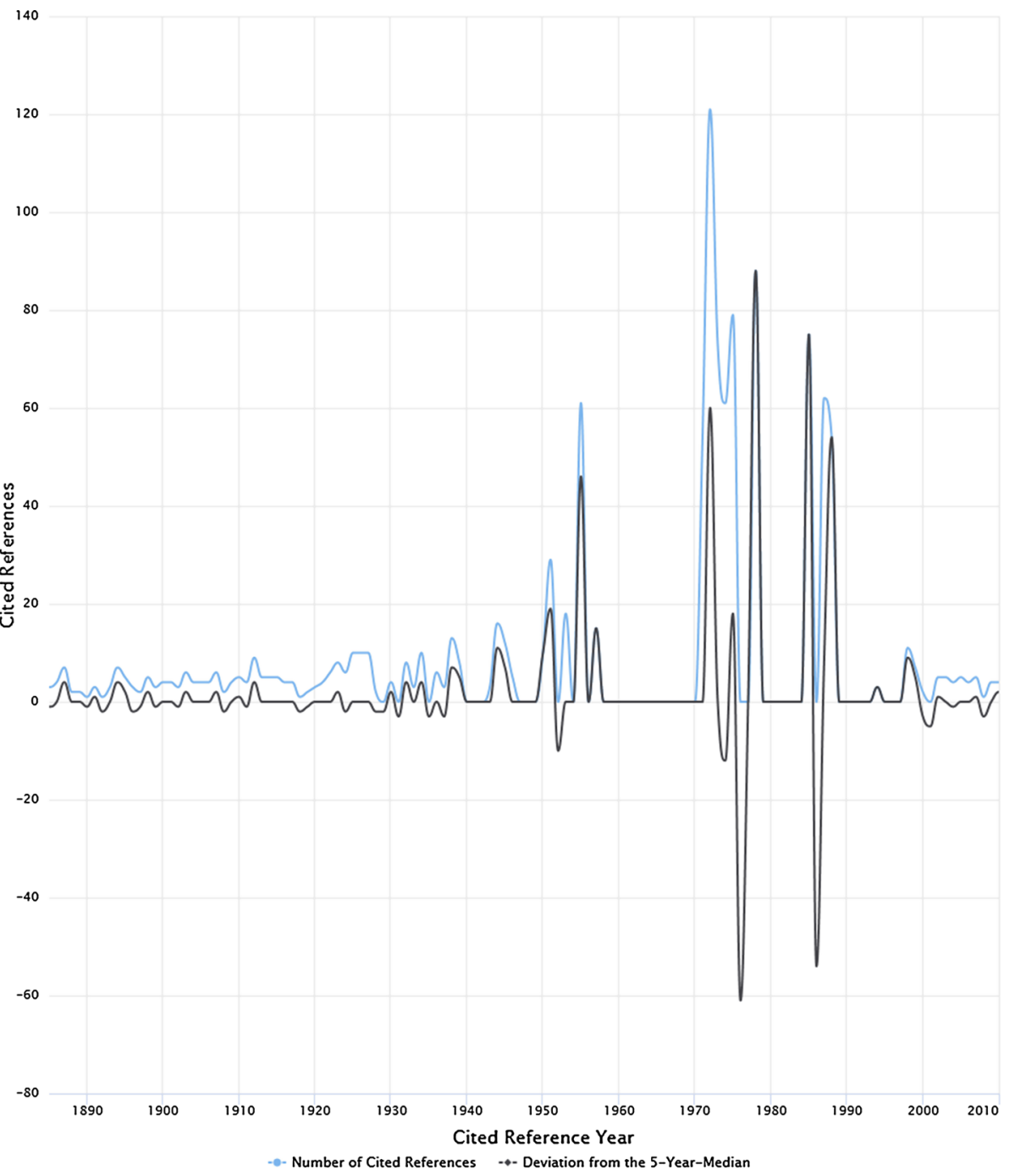

Fig. 2 Spectrogram of data set $1(n=328 \mathrm{CRs})$. The number of CRs (blue line) and the five-year median deviation (black line) are shown

We discuss the other important CRs using Table 3 which shows the CRs with the largest number of occurrences within data set 2 (without self-citations). On the first glance, we can observe significantly lower numbers of occurrences for the CRs. Thus, these publications were less important for EG to write his papers than his own publications. The CRs in Table 3 can be divided into three groups: (1) bibliometrics, (2) biology, and (3) nonscholarly material.

The group of CRs related to bibliometrics is the largest group in Table 3 with five CRs (the fifth, seventh, eighth, ninth, and tenth CR). The group of CRs which belong to the field of biology is only slightly smaller with four CRs (the first, second, fourth, and eleventh $\mathrm{CR}$ ). The smallest group of CRs which belongs to non-scholarly material is composed of 
Table 2 CRs with the largest number of occurrences (including self-citations)

\begin{tabular}{lll}
\hline CR & RPY & $\begin{array}{l}\text { Number of } \\
\text { occurrences }\end{array}$ \\
\hline $\begin{array}{l}\text { Garfield, E., 1955, Citation indexes for science-new dimension in } \\
\text { documentation through association of ideas. Science, 122(3159), 108-111 }\end{array}$ & 195561 \\
Garfield, E., 1971, Current Contents, P5 & 197154 \\
Garfield, E., 1972, Citation analysis as a tool in journal evaluation: journals can & 1972 & 64 \\
be ranked by frequency and impact of citations for science policy studies. & & \\
Science, 178(4060), 471-479 & 1972 & 57 \\
Garfield, E., 1972, Current Contents, 1101, P5 & 1973 & 73 \\
Garfield, E., 1973, Current Contents, P5 & 1974 & 61 \\
Garfield, E., 1974, Current Contents, P5 & 1975 & 79 \\
Garfield, E., 1975, Current Contents, P5 & 1978 & 88 \\
Garfield, E., 1978, Current Contents, P5 & 1985 & 75 \\
Garfield, E., 1985, Current Contents, V43, P3 & 1987 & 62 \\
Garfield, E., 1987, Current Contents, P3 & 1988 & 54 \\
Garfield, E., 1988, Current Contents, P3 & & \\
\hline
\end{tabular}

The CRs which are responsible for the large peaks in Fig. 2 are shown

two CRs (the third and sixth CR). The CRs in Table 3 are ordered by decreasing number of occurrences.

The second and fourth CRs report the discovery (the fourth CR, Avery et al. with RPY $=1944$ ) and the structure (the second CR, Watson and Crick with RPY = 1953) of Deoxyribose Nucleic Acid (DNA). Watson and Crick described the DNA structure as a double helix in the second CR in Table 3. These authors received the Nobel Prize in 1962 for this work together with Maurice Hugh Frederick Wilkins. The eleventh CR (Selye with RPY $=1946$ ) has been referenced by EG three times in Current Contents. EG also used this CR as an example paper in his 1955 paper (first CR of Table 2). His 1955 paper was reprinted in 2003 (see https://doi.org/10.1093/ije/dyl189) and therefore, counts as two references.

The fifth CR (Bradford, RPY $=1950$ ) is foundational for bibliometrics: among other things, it discusses Bradford's (1934) law of scattering. The ninth CR (RPY $=1934)$ is the original publication of Bradford's law of scattering. The seventh CR $(\mathrm{RPY}=1927)$ is the proposal by Gross and Gross (1927) to use the number of times a journal is cited as a criterion for purchasing it in the library. Therefore, it can be seen as a predecessor of the JIF. The eighth CR $(\mathrm{RPY}=1944)$ is a critical assessment of the aforementioned journal selection criterion using physiology journals as an example. Probably, this CR has fueled EG's pursuit towards the JIF. The tenth CR (RPY = 2002) is a paper by Pudovkin and Garfield (2002) where they propose a relatedness factor (RF) for journals calculated from citation counts, number of papers, and number of references. The RF groups journals close together which are also semantically similar.

The third CR (RPY = 1945) is an essay about the tasks of scientists after the Second World War. Many aspects of the information society were discussed in this essay. The sixth CR (RPY = 1938) is the well-known novel "World Brain" by H. G. Wells. 


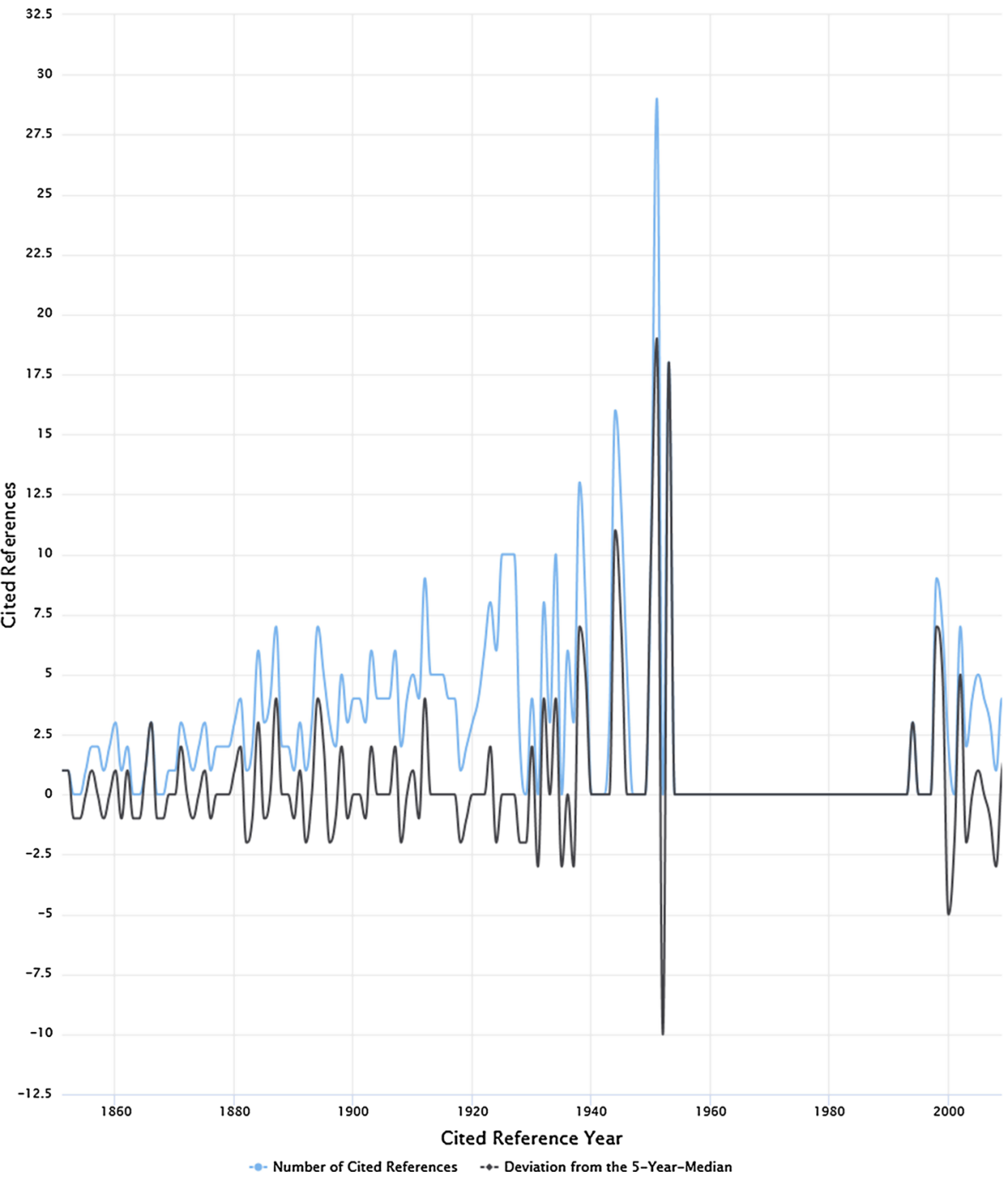

Fig. 3 Spectrogram of data set $2(n=316 \mathrm{CRs})$. The number of CRs (blue line) and the five-year median deviation (black line) are shown

\section{Discussion}

Beyond doubt, EG has been one of the most influential scientists in the field of bibliometrics. The field would perhaps not exist, if EG had not introduced the idea of indexing citations for quantitative analyses in the history and philosophy of science (Elkana et al. 1978; Gingras 2016; Wouters 2000). EG created the fundament on which all empirical studies in bibliometrics are based. In addition to the idea of citation indexing, he initiated several lines of research in bibliometrics later on. For example, the development of indicators based on bibliometric data started with the introduction of the JIF (Garfield 2006; Garfield and Sher 1963). Since this initial introduction, the development of several 
Table 3 CRs with the largest number of occurrences (without self-citations), ordered by decreasing number of occurrences

\begin{tabular}{ll}
\hline $\mathrm{CR}$ & RPY $\begin{array}{l}\text { Number of } \\
\text { occurrences }\end{array}$ \\
\hline
\end{tabular}

Lowry, O. H., Rosebrough, N. J., Farr, A. L., \& Randall, R. J. (1951). Protein

195129

Measurement with the Folin Phenol Reagent. Journal of Biological Chemistry, 193(1), 265-275

Watson, J. D., \& Crick, F. H. C. (1953). Molecular structure of nucleic acids-a structure for Deoxyribose Nucleic Acid. Nature, 171(4356), 737-738

Bush, Vannevar (1945). As we may think. Atlantic Monthly 176, 101-108

Avery, Oswald T., MacLeod, Colin M., \& McCarty, Maclyn. (1944). Studies on the chemical nature of the substance inducing transformation of pneumococcal types. Journal of Experimental Medicine, 79(2), 137-158

Bradford, S. C. (1950). Documentation. Washington, DC, USA: Public Affairs Press

Wells, H.G. (1938). World Brain. London. UK: Methuen

195318

194512

194410

$1950 \quad 10$

19389

Gross, P. L. K., \& Gross, E. M. (1927). College libraries and chemical education. Science, 66, 385-389

Brodman, Estelle. (1944). Choosing Physiology Journals. Bulletin of the Medical Library Association, 32(4), 479-483

Bradford, S. (1934). On the scattering of papers on scientific subjects in scientific periodicals. Engineering, 137(193), 85

Pudovkin, A. I., \& Garfield, E. (2002). Algorithmic procedure for finding semantically related journals. Journal of the American Society for Information Science and Technology, 53(13), 1113-1119

Selye, H. (1946). The general adaptation syndrome and the diseases of adaptation. Journal of Clinical Endocrinology \& Metabolism, 6, 117-231

CRs with at least 5 occurrences are shown

(advanced) indicators followed in bibliometrics which do not only refer to journals (several journal-based indicators can be found in Clarivate Analytics' Journal Citation Report), but also to other entities (e.g., to single researchers with the $h$ index).

EG was the first who formulated a list of possible reasons why documents are cited in publications (Bornmann and Daniel 2008). Thus, he started the discussion in bibliometrics about the meaning of citations and the formulation of underlying theoretical approaches. A further example of his groundbreaking work is the introduction and development of algorithmic historiography (Garfield 2004; Garfield et al. 1964; Leydesdorff 2010). The method which can be applied by using the historiographic software HistCite ${ }^{\mathrm{TM}}$ "facilitates the understanding of paradigms by enabling the scholar to identify the significant works on a given topic" (Garfield et al. 2003, p. 400). Similar software, which has been developed on this base later on, are CitNetExplorer (http://www.citnetexplorer.nl, see van Eck and Waltman 2014) and CRExplorer (www.crexplorer.net, see Thor et al. 2016a, b).

This study deals with EG's roots (studies, theories, and ideas) and how they are reflected in EG's publications. We used the CRExplorer-which has its roots in EG's own software developments-and applied RPYS to his publication set. The results show that EG has cited his own publications in many cases. This can be possibly traced back to the fact that EG was a pioneer not only in shaping the citation index, but also in many lines of research in bibliometrics. Furthermore, inspiring inputs might have been come from non- 
citable sources. For example, Shepard's Citations is a citation index which is used in US legal research. It lists all authorities who cite particular cases, statutes, or other legal authorities. EG later acknowledged that his citation index was heavily influenced by Shepard's Citations (Wouters 1999a). However, this does not reflect in (or cannot be retrieved from) his citations, because there does not exist a paper on Shepard's Citations which could be cited in this context.

Among the papers most referenced by EG are some non-scholarly publications, some publications from the early evolving field of bibliometrics, and publications from other disciplines for the presentation of examples. The heterogeneity of the cited publications demonstrates the wide-spreading interests of EG which range from the historiography of science as an empirical operationalization for questions in the history and philosophy of science (e.g. Elkana et al. 1978; Gingras 2016), citation indexing as an approach to document retrieval (Garfield 1955), journal evaluation for library management (Bradford 1950; Garfield 1957, 1972), the mapping of the sciences (Garfield 1987; Small and Garfield 1985), to bibliometrics and the theory of citation (Garfield 1975, 1979).

The prevailing publication and citation of non-scholarly publications makes clear that EG's interests are not limited by scholarly bounds. They reach beyond the narrow definitions of scientific epistemological interests and perform the translation of the latter into knowledge-based innovations.

Acknowledgements Open access funding provided by Max Planck Society.

Open Access This article is distributed under the terms of the Creative Commons Attribution 4.0 International License (http://creativecommons.org/licenses/by/4.0/), which permits unrestricted use, distribution, and reproduction in any medium, provided you give appropriate credit to the original author(s) and the source, provide a link to the Creative Commons license, and indicate if changes were made.

\section{References}

Bornmann, L., \& Daniel, H.-D. (2008). What do citation counts measure? A review of studies on citing behavior. Journal of Documentation, 64(1), 45-80. https://doi.org/10.1108/00220410810844150.

Bradford, S. (1934). On the scattering of papers on scientific subjects in scientific periodicals. Engineering, $137(193), 85$.

Bradford, S. C. (1950). Documentation. Washington, DC: Public Affairs Press.

Elkana, Y., Lederberg, J., Merton, R. K., Thackray, A., \& Zuckerman, H. (1978). Toward a metric of science: the advent of science indicators. New York, NY: Wiley.

Garfield, E. (1955). Citation indexes for science-new dimension in documentation through association of ideas. Science, 122(3159), 108-111.

Garfield, E. (1957). Breaking the subject index barrier-a citation index for chemical patents. Journal of the Patent Office Society, 39(8), 583-595.

Garfield, E. (1972). Citation analysis as a tool in journal evaluation. Science, 178(4060), 471-479.

Garfield, E. (1975). The 'Obliteration Phenomenon' in science-and the advantage of being obliterated! Current Contents, 51(52), 5-7.

Garfield, E. (1979). Citation indexing-its theory and application in science, technology, and humanities. New York, NY: Wiley.

Garfield, E. (1987). Launching the ISI Atlas of Science: For the new year, a new generation of reviews. Current Contents (1), 3-8.

Garfield, E. (2004). Historiographic mapping of knowledge domains literature. Journal of Information Science, 30(2), 119-145.

Garfield, E. (2006). The history and meaning of the journal impact factor. Journal of the American Medical Association, 295(1), 90-93.

Garfield, E., \& Sher, I. H. (1963). New factors in the evaluation of scientific literature through citation indexing. American Documentation, 14(3), 195-201. 
Garfield, E., Pudovkin, A. I., \& Istomin, V. S. (2003). Why do we need algorithmic historiography? Journal of the American Society for Information Science and Technology, 54(5), 400-412. https://doi.org/10. 1002/Asi.10226.

Garfield, E., Sher, I. H., \& Torpie, R. J. (1964). The use of citation data in writing the history of science. Philadelphia, PA: Institute for Scientific Information.

Gingras, Y. (2016). Bibliometrics and research evaluation: uses and abuses. Cambridge MA: MIT Press.

Gross, P. L. K., \& Gross, E. M. (1927). College libraries and chemical education. Science, 66, 385-389.

Hou, J. (2017). Exploration into the evolution and historical roots of citation analysis by referenced publication year spectroscopy. Scientometrics, 110(3), 1437-1452. https://doi.org/10.1007/s11192-0162206-9.

Leydesdorff, L. (2010). Eugene Garfield and algorithmic historiography: Co-words, co-authors, and journal names. Annals of Library and Information Studies, 57(3), 248-260.

Lowry, O. H., Rosebrough, N. J., Farr, A. L., \& Randall, R. J. (1951). Protein measurement with the Folin phenol reagent. Journal of Biological Chemistry, 193(1), 265-275.

Marx, W., Bornmann, L., Barth, A., \& Leydesdorff, L. (2014). Detecting the historical roots of research fields by reference publication year spectroscopy (RPYS). Journal of the Association for Information Science and Technology, 65(4), 751-764. https://doi.org/10.1002/asi.23089.

Pudovkin, A. I., \& Garfield, E. (2002). Algorithmic procedure for finding semantically related journals. Journal of the American Society for Information Science and Technology, 53(13), 1113-1119. https:// doi.org/10.1002/Asi.10153.

Small, H. (2017). A tribute to Eugene Garfield: Information innovator and idealist. Journal of Informetrics, 11(3), 599-612. https://doi.org/10.1016/j.joi.2017.04.006.

Small, H., \& Garfield, E. (1985). The geography of science-disciplinary and national mappings. Journal of Information Science, 11(4), 147-159. https://doi.org/10.1177/016555158501100402.

Thor, A., Marx, W., Leydesdorff, L., \& Bornmann, L. (2016a). Introducing CitedReferencesExplorer (CRExplorer): A program for reference publication year spectroscopy with cited references standardization. Journal of Informetrics, 10(2), 503-515.

Thor, A., Marx, W., Leydesdorff, L., \& Bornmann, L. (2016b). New features of CitedReferencesExplorer (CRExplorer). Scientometrics, 109(3), 2049-2051.

van Eck, N. J., \& Waltman, L. (2014). CitNetExplorer: A new software tool for analyzing and visualizing citation networks. Journal of Informetrics, 8(4), 802-823. https://doi.org/10.1016/j.joi.2014.07.006.

van Raan, A. F. J., \& Wouters, P. (2017). Eugene Garfield 1925-2017: Visionary information scientist. Retrieved April 26, 2017, from https:/www.cwts.nl/news?article=n-q2y2c4\&title=eugene-garfield1925-2017-visionary-information-scientist.

Wouters, P. (1999a). The citation culture. Unpublished Ph.D. Thesis, University of Amsterdam, Amsterdam

Wouters, P. (1999b). The creation of the Science Citation Index. Medford: Information Today Inc.

Wouters, P. (2000). Garfield as alchemist. In B. Cronin \& H. B. Atkins (Eds.), The web of knowledge: A Festschrift in honor of Eugene Garfield (Vol. ASIS Monograph Series) (pp. 65-71). Medford, NJ: Information Today. 